\title{
Impact of economic factors, social health and stressful life events on physical health-related quality of life trajectories in older Australians
}

\author{
Aung Zaw Zaw Phyo ${ }^{1}$ - David A. Gonzalez-Chica ${ }^{2,3} \cdot$ Nigel P. Stocks $^{2} \cdot$ Robyn L. Woods $^{1} \cdot$ Jane Fisher $^{1} \cdot$ Thach Tran $^{1}$. \\ Alice J. Owen ${ }^{1}$. Stephanie A. Ward ${ }^{1,4}$. Carlene J. Britt ${ }^{1}$. Joanne Ryan ${ }^{1} \cdot$ Rosanne Freak-Poli ${ }^{1,5}{ }^{-1}$ on behalf of the \\ ASPREE Study Group
}

Accepted: 15 October 2021 / Published online: 22 October 2021

(c) The Author(s), under exclusive licence to Springer Nature Switzerland AG 2021

\begin{abstract}
Purpose Physical health-related quality of life (HRQoL) is associated with adverse health outcomes, including hospitalizations and all-cause mortality. However, little is known about how physical HRQoL changes over time in older people and the predictors of this trajectory. This study (a) identified trajectories of physical HRQoL among older people and (b) explored whether economic factors, social health or stressful life events impact physical HRQoL trajectories.

Method A cohort of 12,506 relatively 'healthy' community-dwelling Australians aged $\geq 70$ years ( $54.4 \%$ females), enrolled in the ASPREE Longitudinal Study of Older Persons (ALSOP) study and was followed for six years. Economic factors, social health and life events in the last 12 months were assessed through a questionnaire at baseline. Physical HRQoL was measured by using the 12-item short form at baseline and annual follow-ups. Growth mixture and structural equation modelling were used to identify physical HRQoL trajectories and their predictors.

Results Four physical HRQoL trajectories were identified—stable low (7.1\%), declining (9.0\%), stable intermediate (17.9\%) and stable high (66.0\%). Living in more disadvantaged areas, having a lower household income, no paid work, no voluntary work, loneliness and stressful life events (i.e. spousal illness, friend/family illness, financial problem) were associated with a 10\%-152\% higher likelihood of being in the stable low or declining physical HRQoL trajectory than the stable high group. Conclusion Specific stressful life events had a greater impact on adverse physical HRQoL trajectories in older people than other factors. Volunteering may prevent physical HRQoL decline and requires further investigation.
\end{abstract}

Keywords Physical health-related quality of life · Economic factors · Social health · Stressful life events · Trajectories . Older people

\section{Introduction}

Joanne Ryan and Rosanne Freak-Poli contributed equally as cosenior authors.

The members of ASPREE Investigator Group are listed on http:// www.aspree.org.

Rosanne Freak-Poli

Rosanne.Freak-Poli@monash.edu

1 Department of Epidemiology and Preventive Medicine, School of Public Health and Preventive Medicine, Monash University, 553 St Kilda Road, Melbourne, VIC 3004, Australia

2 Discipline of General Practice, Adelaide Medical School, The University of Adelaide, Adelaide, SA 5005, Australia
The world has an ageing demographic, and globally, the proportion of people aged $\geq 65$ years is projected to more than double from $9.3 \%$ in 2020 to $16.0 \%$ in 2050 [1, 2]. Like other high-income countries, the Australian population is

3 Adelaide Rural Clinical School, The University of Adelaide, Adelaide, SA 5005, Australia

4 Centre for Healthy Brain Ageing, School of Psychiatry, University of New South Wales, Sydney, NSW 2052, Australia

5 Department of Epidemiology, Erasmus Medical Centre, 3015 GD Rotterdam, The Netherlands 
ageing with the proportion aged $\geq 65$ years increasing from $12.4 \%$ in 2000 to $16.3 \%$ in 2020 [3]. However, this increase in life expectancy does not necessarily correspond to an increase in healthy and disability-free years of life among older people $[1,4,5]$. Given that existing health services are not well designed to handle the specific needs of older people, population ageing will increase the burden on the healthcare system around the globe as well as in Australia $[1,6]$. As such, government policies need a stronger focus on the promotion of healthy ageing (i.e. the process of creating environments that will enable older people to live independently and maintain their functional capacities as long as possible) [7] and identifying specific groups at higher risk of adverse ageing-related health outcomes is essential for developing preventive intervention strategies.

Physical health-related quality of life (HRQoL) is a selfreported measure that assesses the impact of physical health status on individuals' daily lives [8]. In health sciences, the physical domain of HRQoL has been increasingly identified as an effective indicator of health outcomes, including disability, falls, cancer, cardiovascular disease (CVD), hospitalizations and all-cause mortality [8-13]. Previous studies have investigated disease-specific HRQoL trajectories in patients affected by chronic diseases such as coronary heart disease or cancer [14, 15]. However, no studies have examined how the physical HRQoL changes over time among relatively healthy older people living in the community, or what determinants may have an adverse effect on physical HRQoL.

The social determinants conceptual framework proposed by the World Health Organization (WHO) considers that health is governed by different structural and intermediary determinants, including socioeconomic conditions, social health and stressful life circumstances [7]. Life-course transitions, such as reduced income after retirement, relocation and bereavement or loss of a spouse/partner are common examples of stressors in older people [16]. The impact of these late-life transitions may play an essential role in determining the HRQoL of older people [17-19]. For example, a retiree may experience financial stress and may need to learn to live on a reduced budget, limiting spending on hobbies, entertainment and social activities. Prior cross-sectional studies have demonstrated that people with low income or a lower socioeconomic position are more likely to have poor HRQoL [20-23]. Furthermore, cross-sectional evidence also showed that some life events like being divorced, experiencing relational problems with friends/relatives or limited social engagements are associated with poor physical HRQoL in older individuals [17, 23, 24]. However, there is limited evidence on whether such events can impact the trajectory of physical HRQoL in later life.

In this study, we aimed to identify (a) trajectories of physical HRQoL among relatively 'healthy' community-dwelling older Australians over time, and (b) whether economic factors, social health or other recent stressful life events (i.e. last 12 months) predict physical HRQoL trajectories.

\section{Methods}

\section{Study population}

This study used longitudinal data from the ASPirin in Reducing Events in the Elderly (ASPREE) study and the ASPREE Longitudinal Study of Older Persons (ALSOP) sub-study, which was conducted in parallel to ASPREE.

Briefly, ASPREE was a double-blind, randomized placebo-controlled trial, which aimed to determine the effect of low-dose aspirin on primary prevention among the Australian and United States (U.S.) people [25]. From 2010 through 2014, ASPREE recruited 19,114 individuals aged $\geq 70$ years (or aged $\geq 65$ years for U.S. minority groups) who were free of serious conditions with a short life expectancy (e.g. cancer, CVD) $[25,26]$. ASPREE participants were followed prospectively until June 12, 2017 [26, 27]. The 14,892 (89\%) of total 16,703 Australian ASPREE participants agree to participate in ALSOP sub-study (Supplementary Figure S1) [28]. Of these, about 12,506 participants completed the social questionnaire at ALSOP baseline, and the HRQoL assessment at the ASPREE baseline and two or more follow-ups (Supplementary Figure S1), representing the target sample of this study.

\section{Outcome: physical health-related quality of life}

The physical HRQoL was assessed at the baseline and over six follow-ups using the validated Medical Outcomes Study 12-item short form (SF-12, version 2; Cronbach's alpha $=0.86$ ) [29]. The physical component score (PCS) of HRQoL was generated using a norm-based scoring algorithm with a mean of 50 and a standard deviation of 10 , with a higher score indicating a better physical HRQoL [30]. Estimation of the PCS considers heavier weighting of physical functioning, role limitation due to physical health, bodily pain and general health domains of SF-12 [30].

\section{Determinants}

Based on the WHO social determinants conceptual framework [7], the hypothesized model was developed and modified to accommodate our research questions and data availability (Fig. 1). All determinants were assessed at baseline. 


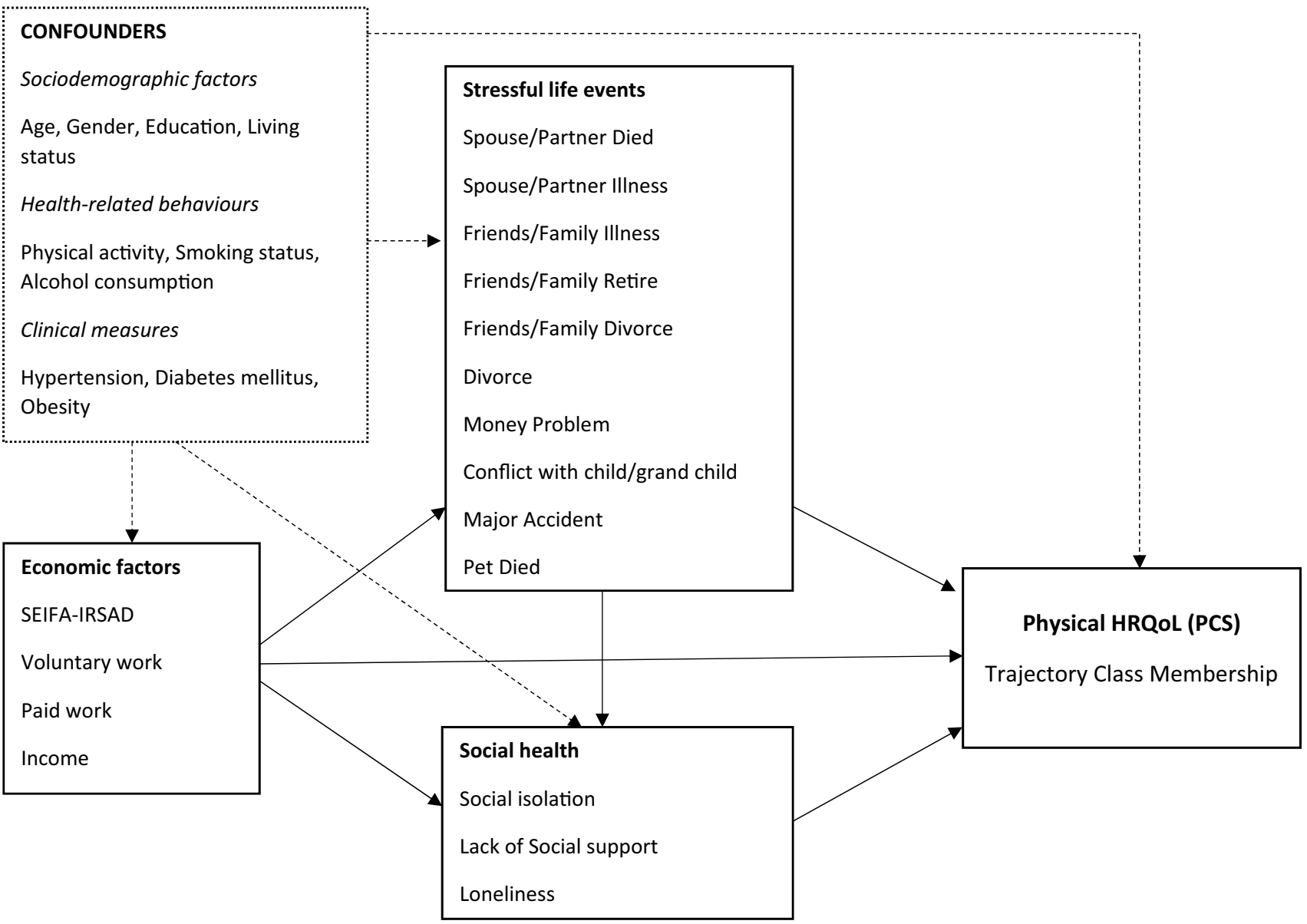

Fig. 1 Hypothesized model of economic factors, social health and life events predicting physical health-related quality of life trajectory class memberships, controlling for sociodemographic factors, healthrelated behaviours and clinical measures. Physical HRQoL physical

\section{Economic factors}

Using the residential postcodes of participants, we also estimated the Socio-Economic Indexes for Areas-Index of Relative Socioeconomic Advantage and Disadvantage (SEIFAIRSAD) based on the information from the 2011 Australian Census [31]. The SEIFA-IRSAD index, a macro-economic indicator of socioeconomic status is the summarized score of the economic and social conditions of people and households within an area [32]. The SEIFA-IRSAD index calculation considers annual household income, education, occupation, internet access and rental rate of dwellings within a relative area [32]. The SEIFA-IRSAD deciles were categorized into quintiles and then used as a continuous variable, with a lower score indicating a person lives in a more disadvantaged area [31, 32].

Other economic factors investigated were (a) household gross income; (b) full-time/part-time paid work (yes or no); and (c) voluntary work, not including child minding, baby health-related quality of life, SEIFA-IRSAD Socio-Economic Indexes for Areas-Index of Relative Socioeconomic Advantage and Disadvantage

sitting or caring (yes or no). Household income was analysed as a binary variable and categorized as, < AU\$50,000 per year or $\geq$ AU $\$ 50,000$ per year (those who preferred not to report their income included in this category).

\section{Social health}

The social health investigated in this study included social isolation, lack of social support and loneliness (Supplementary Table S1). Social isolation and social support were assessed using the Lubben Social Network Scale-Revised (LSNS-R) [33]. The Cronbach's alpha of survey items was 0.77 . Social isolation was defined as engaging in community activities less than once per month and having contact with four or fewer relatives or close friends in a month. Lack of social support was defined as having fewer than four relatives or close friends with whom they can call on for help and discuss private matters. Loneliness was assessed using one of the questions of the Center for Epidemiological 
Studies-Depression (CES-D-10) [34] and defined as feeling lonely for three or more days in the past seven days.

\section{Stressful life events}

Ten stressful life events over the previous 12 months were assessed with yes-no responses, which included (a) death of a spouse/partner, (b) serious illness in a spouse/partner, (c) death or serious illness in family members or close friends, (d) job loss or retirement in family members or close friends, (e) divorce or partnership breakup in family members or close friends, (f) personal divorce or partnership breakup, (g) major problems with money, (h) major conflict with children or grandchildren, (i) major accidents, disasters, muggings, unwanted sexual experiences, robberies or similar events and (j) having a pet that died.

\section{Covariates}

Baseline demographic, lifestyle and clinical factors associated with the physical HRQoL were considered potential confounders [35]. Demographic factors included age in years, gender, years of education and living situation. Lifestyle behaviours consisted of physical activity levels, smoking status and alcohol consumption [36]. Clinical measures included hypertension [26], diabetes [26] and obesity (body mass index $\geq 30.0 \mathrm{~kg} / \mathrm{m}^{2}$ based on measured weight and height) [37]. Depressive symptoms were not adjusted for in the main analysis, given that loneliness was assessed using a question from the CES-D-10 (depression scale) [34] and collinearity between these variables was expected. However, adjustment was performed in sensitivity analysis, removing the loneliness question for the overall depressive symptom score (CES-D-9).

\section{Data analysis}

Analyses were conducted using a three-step approach [38]. In the first step, growth mixture modelling (GMM) was fitted to identify the trajectories (latent patterns of longitudinal change) of PCS over seven time points (i.e. baseline and up to six follow-ups), using the Ram and Grimm's approach [39]. We assessed a series of GMM models that varied in the number of latent patterns and shapes of change (linear, quadratic, or cubic). The final best-fit model was selected using prespecified criteria [39], including (a) whether the model makes sense mathematically (i.e. no negative variances and/or problems in estimation); (b) information criteria: whether Bayesian Information Criteria (BIC), Akaike Information Criteria (AIC) and adjusted BIC model were the lowest scores, which mean the best-fit model for the data; (c) likelihood ratio tests: whether the likelihood ratio tests that assess specific comparisons between the model of interest and a model with one fewer class had p values $<0.05$; (d) whether the trajectories were theoretically justifiable; and (e) using the entropy statistic, how well the final model separated the latent classes. The entropy statistic is a summary indicator of the conditional probabilities of an individual's group membership, and it ranges from 0.00 (low) to 1.00 (high), with a higher value indicating that individuals are classified with greater confidence [40].

In the second step, each individual was assigned to a trajectory class using the latent class posterior probability distribution of our final GMM model [38]. This assigned trajectory was used as the primary outcome variable, with the highest PCS trajectory as the reference group.

In the third step, structural equation modelling (SEM) was used to investigate whether economic factors, social health and having stressful life events predict each PCS trajectory membership [38]. The model was specified based on Fig. 1. Further, sensitivity analyses were performed. The final model was repeated using the categorical income (<AU\$20,000/year, AU\$20,000-49,999/year, AU\$50,000-99,999/year, $\geq$ AU\$100,000/year) as a continuous variable (in which preferred not to answer included as a middle-income category). The final model was also repeated after excluding individuals who died during the follow-up period and lastly after adjustment for CES-D-9 score. All analyses were undertaken using Mplus version 8.6 (Muthén \& Muthén, Los Angeles, U.S.).

\section{Results}

The present study included 12,506 Australians aged $\geq 70$ years (mean age $75.2 \pm 4.3$ years; $54.4 \%$ females). Table 1 shows that at the baseline, most participants $h a d \geq 12$ years of education, lived with someone else, had an income of $<$ AU $\$ 50,000$ per year, had positive lifestyle behaviours, hypertension, fewer comorbidities and had not experienced the ten measured stressful life events in the past 12 months.

The fit statistics for growth mixture models of longitudinal physical HRQoL (i.e. PCS) data were shown in supplementary table S2. According to our prespecified criteria, the four-class linear model was the best fit for these PCS data. Over time, most of the participants had good PCS and all trajectories except one with a declining trend remained stable in each trajectory. The identified class for PCS trajectories was as follow: a stable low PCS $(n=891,7.1 \%)$, a declining PCS $(n=1125,9.0 \%)$, a stable intermediate PCS $(\mathrm{n}=2240,17.9 \%)$ and a stable high PCS $(\mathrm{n}=8250,66.0 \%)$ class (Fig. 2). 
Table 1 Baseline characteristics of participants $(\mathrm{N}=12,506)$
Characteristics

Frequency $(\%)$

or mean $\pm \mathrm{SD}$

\begin{tabular}{|c|c|}
\hline \multicolumn{2}{|l|}{ Sociodemographic factors } \\
\hline Age in years (range: 70-95) & $75.2 \pm 4.3$ \\
\hline Women (vs men) & $6801(54.4 \%)$ \\
\hline Education $12+$ years $(\mathrm{vs}<12$ years) & $6482(51.8 \%)$ \\
\hline Living with someone (vs living alone) & $9085(72.7 \%)$ \\
\hline \multicolumn{2}{|l|}{ Economic factors } \\
\hline \multicolumn{2}{|l|}{ SEIFA-IRSAD } \\
\hline Least advantaged & $1994(15.9 \%)$ \\
\hline 2nd quintile & $2091(16.8 \%)$ \\
\hline 3rd quintile & $2320(18.6 \%)$ \\
\hline 4th quintile & $2395(19.2 \%)$ \\
\hline Most advantaged & $3676(29.5 \%)$ \\
\hline Income $<\$ 50,000$ per year $\left(\right.$ vs others $\left.^{\mathrm{a}}\right)$ & $7986(67.4 \%)$ \\
\hline No full-time/part-time paid work (vs yes) & $11,030(90.8 \%)$ \\
\hline No voluntary work (vs yes) & $7031(57.4 \%)$ \\
\hline \multicolumn{2}{|l|}{ Social health } \\
\hline Social isolation (vs no) & $216(1.9 \%)$ \\
\hline Lack of social support (vs no) & $233(1.9 \%)$ \\
\hline Loneliness (vs no) & $625(5.0 \%)$ \\
\hline \multicolumn{2}{|l|}{ Stressful life events in 12 months prior to baseline } \\
\hline Spouse/partner died (vs no) & $361(3.0 \%)$ \\
\hline Spouse/partner illness (vs no) & $2259(19.2 \%)$ \\
\hline Friends/family illness (vs no) & $5308(43.6 \%)$ \\
\hline Friends/family retire (vs no) & $1625(13.2 \%)$ \\
\hline Friends/family divorce (vs no) & $1767(14.4 \%)$ \\
\hline Divorce (vs no) & $259(2.1 \%)$ \\
\hline Money problem (vs no) & $501(4.1 \%)$ \\
\hline Conflict with child/grandchild (vs no) & $667(5.4 \%)$ \\
\hline Major accident (vs no) & $331(2.7 \%)$ \\
\hline Pet died (vs no) & $1165(9.5 \%)$ \\
\hline \multicolumn{2}{|l|}{ Health-related behaviours } \\
\hline Physical activities rarely/never/light (vs moderate/vigorous) & $4131(33.6 \%)$ \\
\hline Former/current smoking (vs never) & $5477(43.8 \%)$ \\
\hline High risk alcohol consumption (vs never/former/low risk) & $3179(25.4 \%)$ \\
\hline \multicolumn{2}{|l|}{ Clinical measures } \\
\hline Hypertension $^{\mathrm{b}}$ (vs no) & $9291(74.3 \%)$ \\
\hline Diabetes $^{\mathrm{c}}$ (vs no) & $1175(9.4 \%)$ \\
\hline Obesity ${ }^{\mathrm{d}}$ based on BMI (vs no) & $3459(27.8 \%)$ \\
\hline Depression score $^{\mathrm{e}}$ (range: 0-27) median (interquartile range) & $2(1-4)$ \\
\hline
\end{tabular}

SEIFA-IRSAD Socio-Economic Indexes for Areas-Index of Relative Socioeconomic Advantage and Disadvantage

${ }^{\mathrm{a}}$ Other; $\geq \$ 50,000$ per year $(\mathrm{n}=2638,22.3 \%)$ or preferred not to answer $(\mathrm{n}=1228,10.4 \%)$; ${ }^{\mathrm{b}}$ Hypertension $=\mathrm{SBP} \geq 140 \mathrm{mmHg}$ or $\mathrm{DBP} \geq 90 \mathrm{mmHg}$ and/or on treatment for high blood pressure; ${ }^{\mathrm{c}}$ Diabetes mellitus $=$ self-report of diabetes or fasting glucose $\geq 126 \mathrm{mg} / \mathrm{dL}$ or on treatment for diabetes; ${ }^{\mathrm{d}}$ Obesity = body mass index (BMI) $\geq 30$ according to WHO classification; ${ }^{e}$ exclusion of the loneliness item in sum of Center for Epidemiological Studies-Depression (CES-D-10) calculation 


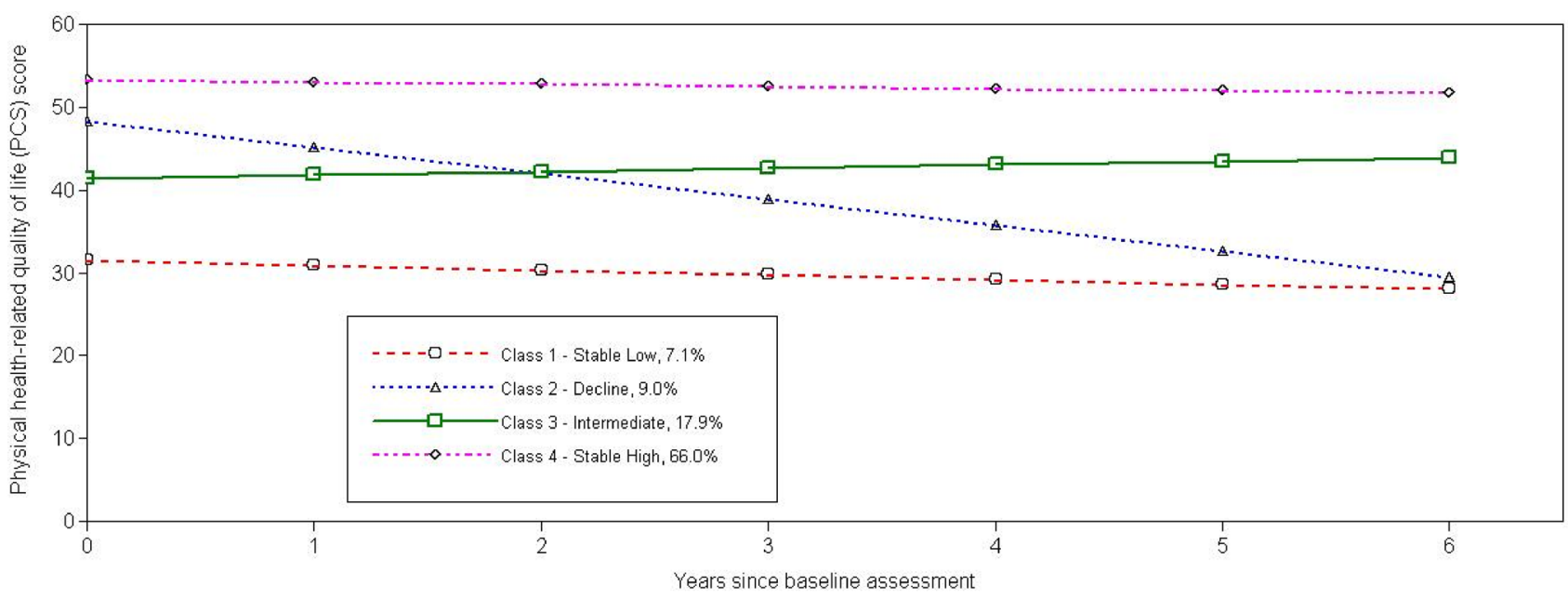

Fig. 2 Physical health-related quality of life trajectory among Australians aged 70+years from baseline (assessment 1) to follow-up 6 years (assessment 7), $\mathrm{N}=12,506$

Table 2 Economic factors, social health and life events predicting physical healthrelated quality of life trajectory class memberships $(\mathrm{N}=11,112)$

\begin{tabular}{|c|c|c|c|}
\hline Comparator: stable high & $\begin{array}{l}\text { Intermediate } \\
\text { OR }(95 \% \mathrm{CI})^{\mathrm{b}}\end{array}$ & $\begin{array}{l}\text { Stable low } \\
\text { OR }(95 \% \mathrm{CI})^{\mathrm{b}}\end{array}$ & $\begin{array}{l}\text { Decline } \\
\text { OR }(95 \% \mathrm{CI})^{\mathrm{b}}\end{array}$ \\
\hline \multicolumn{4}{|l|}{ Economic factors } \\
\hline SEIFA-IRSAD ${ }^{\mathrm{a}}$-lower & $1.06(1.00-1.12)$ & $1.10(1.03-1.17)$ & $1.11(1.03-1.20)$ \\
\hline Income less than $\$ 50,000$ per year-yes & $1.09(0.92-1.30)$ & $1.24(0.98-1.57)$ & $1.35(1.06-1.73)$ \\
\hline Paid work-no & $1.54(1.13-2.09)$ & $2.42(1.45-4.04)$ & $1.60(1.00-2.57)$ \\
\hline Voluntary work-no & $1.06(0.91-1.23)$ & $1.42(1.16-1.74)$ & $1.40(1.13-1.72)$ \\
\hline \multicolumn{4}{|l|}{ Social health } \\
\hline Social isolation-yes & $1.36(0.78-2.39)$ & $1.19(0.61-2.31)$ & $1.24(0.59-2.63)$ \\
\hline Lack of social support-yes & $1.44(0.83-2.49)$ & $1.50(0.81-2.79)$ & $1.38(0.69-2.76)$ \\
\hline Loneliness-yes & $1.12(0.79-1.61)$ & $1.64(1.16-2.32)$ & $1.23(0.81-1.89)$ \\
\hline \multicolumn{4}{|c|}{ Stressful life events in 12 months prior to baseline } \\
\hline Spouse/partner died-yes & $0.90(0.57-1.43)$ & $0.48(0.25-0.90)$ & $0.95(0.54-1.66)$ \\
\hline Spouse/partner illness-yes & $1.07(0.88-1.32)$ & $1.38(1.08-1.76)$ & $1.22(0.94-1.59)$ \\
\hline Friends/family illness_-yes & $1.11(0.95-1.30)$ & $1.32(1.08-1.60)$ & $1.45(1.18-1.78)$ \\
\hline Friends/family retire—yes & $1.20(0.97-1.49)$ & $1.08(0.82-1.42)$ & $1.15(0.86-1.53)$ \\
\hline Friends/family divorce-yes & $1.25(1.01-1.53)$ & $0.78(0.57-1.07)$ & $1.19(0.91-1.57)$ \\
\hline Divorce-yes & $0.75(0.43-1.30)$ & $1.22(0.71-2.10)$ & $0.48(0.18-1.26)$ \\
\hline Money problem-yes & $1.50(1.01-2.22)$ & $2.52(1.74-3.66)$ & $1.89(1.24-2.88)$ \\
\hline Conflict with child/grandchild-yes & $0.97(0.68-1.39)$ & $1.17(0.80-1.71)$ & $1.02(0.65-1.60)$ \\
\hline Major accident—yes & $1.33(0.89-1.97)$ & $1.51(0.93-2.46)$ & $1.07(0.56-2.04)$ \\
\hline Pet died-yes & $1.11(0.86-1.44)$ & 1.49 (1.12-1.97) & $1.24(0.89-1.71)$ \\
\hline
\end{tabular}

SEIFA-IRSAD Socio-Economic Indexes for Areas- Index of Relative Socioeconomic Advantage and Disadvantage

Bold text, refers to having an association between the related factor and PCS trajectory; ${ }^{\text {acategorical }}$ SEIFA-IRSAD variable was treated as a continuous variable; badjusted for age, gender, education, living with someone, physical activities, smoking status, alcohol consumption, hypertension, diabetes and obesity 


\section{Predictors of physical HRQoL (PCS) trajectory}

\section{Economic factors}

Table 2 shows lower area-level socioeconomic status (SEIFA-IRSAD) was associated with a $10 \%-11 \%$ higher likelihood of being in the stable low or decline PCS trajectory than the high stable. No paid work and no voluntary work were associated with up to $142 \%$ higher likelihood of being in the stable low PCS trajectory. However, no voluntary work was also associated with a $40 \%$ higher likelihood of being in the declining PCS trajectory. In contrast, no paid work was associated with a 54\% higher risk of being in the intermediate rather than the high stable PCS trajectory. Lower income was only associated with a $35 \%$ higher likelihood of being in the declining PCS trajectory.

\section{Social health}

Table 2 also shows that loneliness was associated with a $64 \%$ higher likelihood of being in the stable low compared to the high stable PCS trajectory. None of the other social health measures was associated with the trajectory of PCS.

\section{Stressful life events}

Among the stressful life events, the only two conditions associated with a higher likelihood of being either in the decline or stable low PCS trajectories were money problems (up to 2.52 times higher) and friends/family illness (up to 45\% higher; Table 2). Additionally, experiencing spousal/ partner illness and death of a pet were associated with 38\% to $49 \%$ higher likelihood of being in a stable low PCS trajectory. Further, experiencing a spousal/partner death was associated with a $52 \%$ decreased risk of being in a stable low PCS trajectory.

\section{Mediating factors}

Most economic factors had an indirect effect on the PCS trajectory, either through loneliness and/or stressful life events (especially spousal/partner illness) (Fig. 3 and Supplementary Tables S3-S5).

Spousal death, money problems and conflict with children or grandchildren were additional mediating factors between economic variables and the PCS trajectory. Moreover, the relationship between these three stressful life events and the PCS trajectory was mediated by loneliness, either totally

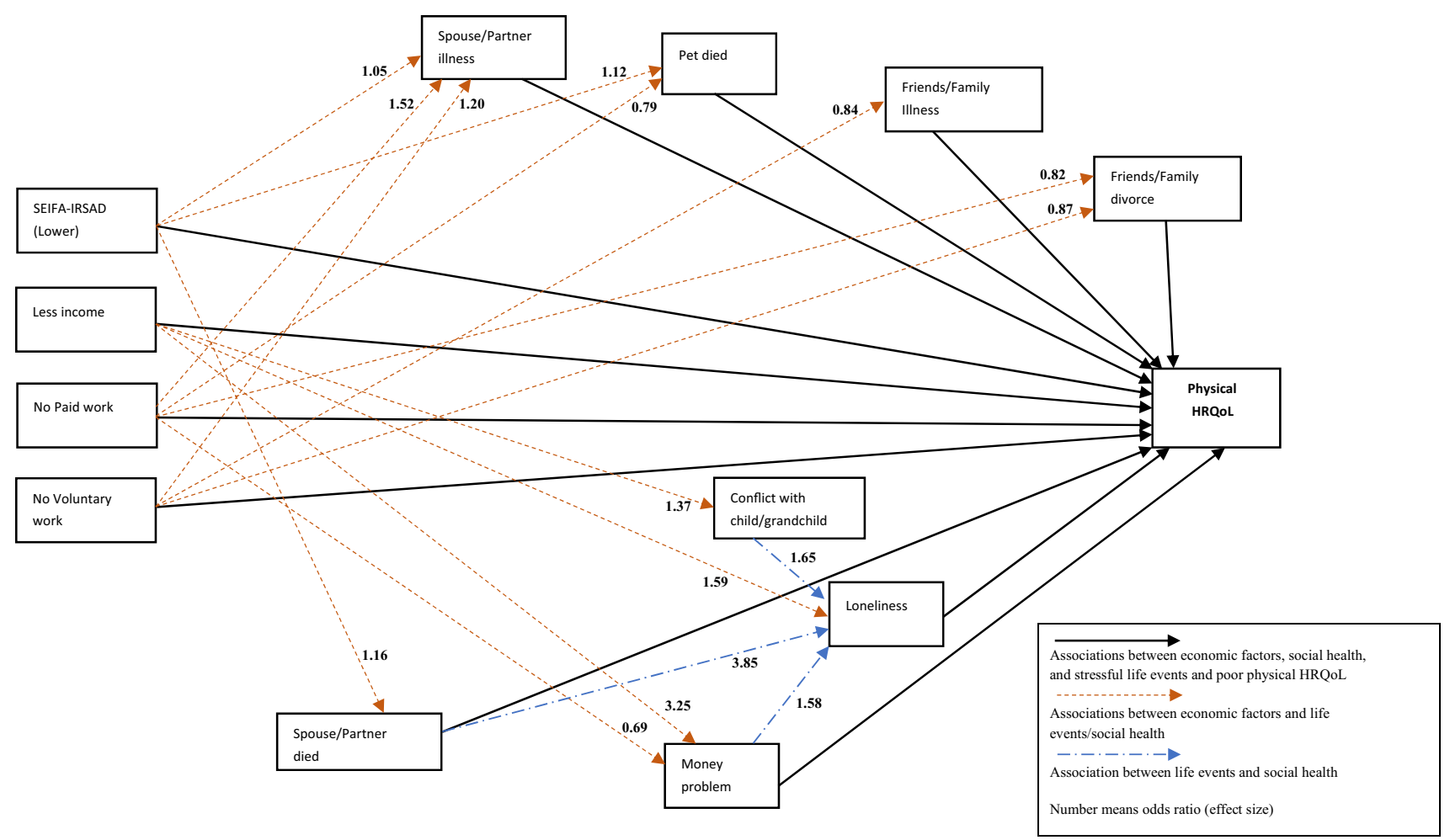

Fig. 3 Associations between economic factors, social health, stressful life events and physical health-related quality of life trajectory class membership. Physical HRQoL physical health-related quality of life,
SEIFA-IRSAD Socio-Economic Indexes for Areas- Index of Relative Socioeconomic Advantage and Disadvantage 
(conflict with child or grandchild) or partially (spousal death, spousal/partner illness and money problem).

The other four stressful life events associated with the PCS trajectory (i.e. spousal/partner illness, illness of friends/ family, divorce of friends/family, death of a pet) had only direct effects on the outcome.

\section{Sensitivity analyses}

The observed associations were consistent with the main findings when income was used as a continuous variable (Supplementary Table S6) and after excluding individuals who died over the study follow-up (Supplementary Table S7). Moreover, the inclusion of CES-D-9 as an additional covariate in the final model decreased the effect size of some associations (specifically loneliness, spousal/ partner illness and friends/family divorce) (Supplementary Table S8).

\section{Discussion}

This study identified different trajectories of physical HRQoL in a large community-based sample of relatively 'healthy' older Australians. Two thirds of the cohort maintained high physical HRQoL levels over the six years of the study. Nonetheless, almost two out of ten older individuals had a persistently low or declining physical HRQoL. These adverse physical HRQoL trajectories were more likely among individuals recently affected by economic, social or stressful life events, especially not undertaking paid work, money problems, loneliness and illness of family/friends. Conversely, voluntary work was an activity practised by $43 \%$ of older adults in this cohorts that seemed to prevent physical HRQoL decline.

The present study identified that not undertaking paid work and money problems were the two variables with the biggest impact on adverse physical HRQoL trajectories in older people. Our finding substantiates the evidence of previous studies showing that employment and financial security play an essential role in maintaining older individuals' overall quality of life (QoL) [41-44]. Further, our mediation analysis found that these relationships were mainly mediated through loneliness or some life events such as spousal/ partner illness, which adds a new finding to the existing literature [41-44]. Moreover, our longitudinal study showed that low income or living in a more disadvantaged area predicted an increased risk of physical HRQoL decline over time. This finding also aligns with previous cross-sectional studies concluding that lower socioeconomic status is associated with poor physical HRQoL in older people [20, 22]. In addition to individual indicators of economic status, an Australian survey revealed that macro-economic indicators of relative socioeconomic disadvantage are also associated with physical HRQoL [21]. Therefore, our research, together with previous literature, highlight that higher economic status is advantageous for maintaining better physical HRQoL in older people.

In addition, we observed that people undertaking volunteer work were more likely to maintain a high physical HRQoL over time. This is supported by existing literature showing that engagement in volunteering can promote overall wellbeing $[42,45]$. A potential mechanism linking volunteering with better QoL may be related to proactive behavioural adaptations and increased social engagement which can complement and may foster self-efficacy, thereby contributing to having a sense of purpose and maintaining better QoL [46]. Most studies, however, mainly explored the impact of volunteering on overall quality of life or psychological wellbeing $[45,46]$. Therefore, our observation demonstrating an association with physical HRQoL specifically, adds novel evidence to the field. Interestingly, we also found that this relationship is mainly meditated by spousal/partner illness. This finding reflects the situation where individuals providing informal care for their chronically ill spouse/ partner might experience limited time for themselves and to engage socially [47].

In terms of social health characteristics, our study demonstrated that loneliness was the only variable predicting persistent low physical HRQoL. Our loneliness finding is in line with previous cross-sectional studies using the SF-12 PCS measure in community-dwelling people aged $\geq 70$ years living in six European countries [48, 49]. They also found that having emotional and social loneliness was associated with a lower physical HRQoL [48, 49]. The potential mechanism linking loneliness with physical HRQoL could involve biological pathways such as diminished immunity, neuroendocrine dysregulation and reduced levels of protective hormones [50]. In particular, loneliness is strongly associated with poor quality of sleep which may have effects on immune, cardiovascular, endocrine and nervous systems. This in turn, could impact general wellbeing and physical health [50]. Furthermore, we observed loneliness as a strong mediator of the associations between money problems, low income or not undertaking paid work and physical HRQol. This finding reflects the existing literature showing the link between economic factors and social health among older individuals, for example, individuals with financial difficulties are less likely to be able to participate in society [51].

Previous studies reported conflicting findings regarding social support/relationship and physical HRQoL in older people [52-54]. In our study, we also found no evidence of associations between either social isolation or social support and physical HRQoL, however the magnitudes of the odds ratios indicate a potential effect. Hence, these null associations could be possibly due to the low prevalence 
(i.e. less than 5\%) of social isolation and lack of social support in our relatively healthy sample, affecting study power. Further research in population with greater social isolation and social support variability is needed to investigate this association.

We provide additional evidence that stressful life events, particularly spousal/partner and friends/family illness, were associated with the risk of being either in the decline or stable low physical HRQoL trajectory. This finding aligns with a recent qualitative Swedish study that found individuals living with a chronically ill spouse/partner were physically exhausted [47]. Further, another noteworthy finding is that spousal/partner illness was the most common mediator of the associations between economic characteristics and physical HRQoL trajectories. These findings may be expected given that managing the chronic illness of a family member imposes an economic impact and usually requires the provision of informal care, creating a ripple effect on the wellbeing of caregivers and family members $[55,56]$. In some cases, families are even required to move to less expensive areas to balance the out-of-pocket costs of looking after a chronically ill family member, increasing the impact on QoL $[55,57]$.

Interestingly, we also found that experiencing a spousal/ partner death was associated with a decreased risk of poor physical HRQoL. This contrasts with prior findings, which show that spousal loss is one of the most stressful life events that affect everyday life [58] and is strongly associated with physical health impairment and mental disorder among recently bereaved spouses [59]. However, the extent to which spousal loss affects the physical wellbeing of widowed persons may vary based on the time after the loss and the burden associated with caring for a chronically ill spouse before death [60]. For example, a study with 1402 Swedish older people aged $\geq 60$ years found that losing a spouse has only a small negative impact on life satisfaction, and this relationship reduced over time [61]. So, individuals may learn healthy coping skills or pursue a volunteer role to promote or resume their social interaction and physical activity after recovering from spousal loss [62].

\section{Implications}

Our findings highlight that cross-sectoral policies through grassroots approaches are required to emphasize economic autonomy, social support networks and coping skills in order to support the physical wellbeing of older people. This suggestion also aligns with the Australian Government's existing social services for older people including age pension, work bonus (i.e. opportunities to receive financial benefits from both work and age pension), seniors card scheme (i.e. offering transport concession to stay connected), free access to broadband internet and personalized mentoring for digital literacy, etc. [63]. Perhaps these existing social services in Australia reflect our results showing most older Australians maintained good physical HRQoL over time. Moreover, within this study, we found a positive impact of volunteer work on the physical wellbeing of older people. Therefore, volunteering programmes could perhaps be considered further as an external social activity which might help reduce the risk of loneliness [51]. Further, our study also identified that experiencing spousal/partner death had the highest risk of loneliness, highlighting the need for intervention.

The current COVID-19 pandemic presents challenges for social interventions such as volunteering, particularly as physical distancing is one of the optimal protective practices to slow the spread of infection [64]. Hence, further innovative technology-based interventions are required, that can increase social interactions through online forums and activities [65].

\section{Strengths}

To the best of our knowledge, our study is the first and largest study exploring the physical HRQOL trajectories in a large cohort of community-dwelling older people with good representation of across the socioeconomic spectrum [28]. Moreover, considering that each life event has its unique effect and severity, our study explored how each of ten adverse life events during the past year rather than the summarized scores predicts the physical HRQoL trajectory. Additionally, we used multi-faceted rather than onedimensional definitions of social isolation and social support (e.g. exclusively focusing on social network size as social isolation).

\section{Limitations}

This study has some limitations. Given that ALSOP recruited relatively 'healthy' Australian participants mainly through general practices [25], our study sample was not intended to be representative of all Australian older people. However, our sample includes an approximately equal proportion of men and women, from noninstitutionalized settings and, fortunately has a diversity in socioeconomic background. Therefore, despite not being representative, it includes good diversity and the results can be extrapolated to healthy older adults who are increasingly being seen in the primary healthcare setting [28]. Moreover, given that a combination of both direct single-item and indirect multi-item scale of loneliness is required to cover a comprehensive picture of loneliness $[66,67]$, we acknowledged that our single-item loneliness scale and our definition based on 'feeling lonely for $\geq 3$ of past 7 days' was not optimal. However, the single-item 
loneliness scale has been acknowledged as valid and seems to be more appropriate for older people [66-68]. Furthermore, social health variables and six of ten stressful life events investigated in this study had a prevalence lower than $10 \%$, affecting the power of the study to investigate these associations. Nonetheless, the sample was large enough to demonstrate that factors even with relatively low frequency, such as loneliness $(5.0 \%)$ or money problems (4.1\%), had an adverse impact on the physical HRQoL trajectories. Additionally, our determinants were mainly measured by using the self-reports which are inherently biased by the individual's feeling at the time of survey completion. For example, individuals who thought their health was poorer, would respond more negative (i.e. loneliness in this study), which may result in our investigated associations being stronger than they truly are. However, the nature of other determinants such as economic factors and life events are not largely related to personal perception, and social isolation and social support were assessed using the validated LSNS-R tool [33] to minimize the selfreport bias. Finally, we acknowledge the limitation that only $75 \%$ of the total Australian ASPREE participants were included in this analysis. However, between included and excluded participants, we generally observed statistically significant differences with small effect sizes for demographic characteristics (Supplementary Table S9). The main difference was for education as included participants being more highly educated $(51.8 \%$ had $\geq 12$ years) than excluded participants $(43.4 \%$, p value $<0.001)$.

\section{Conclusion}

Our study followed longitudinal changes in the physical HRQoL of older individuals for six years and identified that most older adults maintain their good physical HRQoL over time, although some experience a persistently low or declining physical HRQoL. Older individuals who experience economic burden, loneliness or stressful life events are more likely to experience an adverse physical HRQoL trajectory. Additionally, this study also supports prior evidence that provision of volunteering work programmes for older people living in the community may prevent further physical HRQoL decline, reduce age-related healthcare burdens and benefit society. Further research is needed to explore which kinds of voluntary work have the greatest positive impact on physical HRQoL.

Supplementary Information The online version contains supplementary material available at https://doi.org/10.1007/s11136-021-03021-x.
Acknowledgements We would like to thank the ASPREE and ALSOP participants who volunteered for this study, the general practitioners and staff of the medical clinics who support the study participants and the trial staff and management team of the ASPREE study and ALSOP sub-study in Australia and the United States (www.aspree.org).

Author contributions RLW designed and conceptualized the ASPREE study. NPS provided major role in the health-related quality of life component of ASPREE study. SAW, CJB and AJO provided major roles in the acquisition of ALSOP data. RFP and JR conceived the current study. AZZP had full access to all the data in the study and analysed the data. AZZP, RFP and JR interpreted the data, with input from DAGC, JF, TT and AJO. AZZP wrote the initial manuscript draft and undertook revisions. All authors provided critical comments and approved the final version.

Funding ASPREE was supported by grants from the National Institute on Aging and the National Cancer Institute at the U.S. National Institutes of Health (Grant Nos. U01AG029824 and U19AG062682); the National Health and Medical Research Council of Australia (Grant Nos. 334047 and 1127060); Monash University (Australia) and the Victorian Cancer Agency (Australia). ALSOP was supported by funding from Monash University, ANZ Trustees, the Wicking Trust, and the Mason Foundation. Other funding resources and collaborating organizations of the ASPREE study and ALSOP sub-study are listed on http:// www.aspree.org. AZZP is supported by Monash International Tuition Scholarship (Medicine, Nursing and Health Sciences) and Monash Graduate Scholarship (Grant No. 30072360). JF holds the Finkel Chair in Global Health which is supported by the Finkel Family Foundation. JR is supported by a National Health and Medical Research Council Dementia Research Leader Fellowship (Grant No. 1135727). RFP is supported by a National Heart Foundation of Australia Postdoctoral Fellowship (Grant No. 101927). Funders played no role in the design of the study, in the collection, analysis and interpretation of data and in the writing of the manuscript.

Data availability All individual participant data (re-identifiable) that underlie the results reported in this manuscript, are available upon request to qualified researchers without limit of time, subject to approval of the analyses by the Principal Investigators and a standard data sharing agreement. Details regarding requests to access the data will be available through the web site (www.ASPREE.org). The data will then be made available through a web-based data portal safe haven at Monash University, Australia.

\section{Declarations}

Conflict of interest The authors declare that they have no conflict of interest.

Ethical approval ASPREE (ASPirin in Reducing Events in the Elderly) trial and ALSOP (ASPREE Longitudinal Study of Older Persons) sub-study are being conducted in accordance with the Declaration of Helsinki 1964 as revised in 2008, the NHMRC Guidelines on Human Experimentation, the federal patient privacy (HIPAA) law and ICHGCP guidelines and the International Conference of Harmonization Guidelines for Good Clinical Practice. ASPREE and ALSOP also follow the Code of Federal Regulations as it relates to areas of clinical research. The overall management and conduct of the ASPREE clinical trial and ALSOP sub-study are the responsibility of the ASPREE and ALSOP Steering Committee. The data of the present secondary dataanalysis study were from a 5-year ASPREE clinical trial and ALSOP sub-study [Trial Registration: International Standard Randomized Controlled Trial Number Register (ISRCTN 83772183) and clinicaltrials. 
gov (NCT 01038583)]. The current secondary data analysis has been approved by the Monash University Human Research Ethics Committee (Project ID: 21714) and ALSOP received ethics approval from this committee (Social ALSOP: CF11/1935-2011001094). The ASPREE trial was approved by multiple Institutional Review Boards in Australia and the U.S. (www.aspree.org).

Informed consent This study used data from a five-year ASPREE (ASPirin in Reducing Events in the Elderly) clinical trial and ALSOP sub-study (Trial Registration: International Standard Randomized Controlled Trial Number Register (ISRCTN 83772183) and clinicaltrials. gov (NCT 01038583)). All individual participants of the ASPREE clinical trial and ALSOP sub-study signed informed consent on participation.

Research involving human participants ASPREE and ALSOP are being conducted in accordance with the Declaration of Helsinki 1964 as revised in 2008, the NHMRC Guidelines on Human Experimentation, the federal patient privacy (HIPAA) law and ICH-GCP guidelines and the International Conference of Harmonization Guidelines for Good Clinical Practice. ASPREE and ALSOP also follow the Code of Federal Regulations as it relates to areas of clinical research. The overall management and conduct of the ASPREE clinical trial and ALSOP sub-study are the responsibilities of the ASPREE and ALSOP Steering Committee (www.aspree.org).

Consent to participate This study used data from a five-year ASPREE (ASPirin in Reducing Events in the Elderly) clinical trial and ALSOP sub-study [Trial Registration: International Standard Randomized Controlled Trial Number Register (ISRCTN 83772183) and clinicaltrials. gov (NCT 01038583)]. All individual participants of the ASPREE clinical trial and ALSOP sub-study signed informed consent on participation.

Consent for publication All authors gave their approval for submission.

\section{References}

1. He, W., Goodkind, D., Kowal, P., \& Census Bureau, U. S. (2016). An aging world: 2015. Government Publishing Office.

2. United Nations, Department of Economic and Social Affairs \& Population Division. (2020). World Population Ageing 2020 Highlights: Living arrangements of older persons (ST/ESA/ SER.A/451). New York: United Nations.

3. Australian Bureau of Statistics. (2020). Twenty years of population change. Retrieved August 24, 2021, from https://www.abs. gov.au/articles/twenty-years-population-change.

4. Salomon, J. A., Wang, H., Freeman, M. K., Vos, T., Flaxman, A. D., Lopez, A. D., et al. (2012). Healthy life expectancy for 187 countries, 1990-2010: A systematic analysis for the Global Burden Disease Study 2010. The Lancet, 380(9859), 2144-2162.

5. Australian Institute of Health and Welfare. (2018). Older Australia at a glance. Cat. No. AGE 87. Canberra: Australian Institute of Health and Welfare.

6. Australian Institute of Health and Welfare. (2014). Australia's health 2014. Australia's health series No. 14. Cat. No. AUS 178. Canberra: Australian Institute of Health and Welfare.

7. Solar, O., \& Irwin, A. (2010). A conceptual framework for action on the social determinants of health. Social Determinants of Health Discussion Paper 2 (Policy and Practice). Geneva: World Health Organization.
8. Ebrahim, S. (1995). Clinical and public health perspectives and applications of health-related quality of life measurement. Social Science \& Medicine, 41(10), 1383-1394.

9. Truthmann, J., Mensink, G. B. M., Bosy-Westphal, A., Hapke, U., Scheidt-Nave, C., \& Schienkiewitz, A. (2017). Physical healthrelated quality of life in relation to metabolic health and obesity among men and women in Germany. Health and Quality of Life Outcomes, 15(1), 122.

10. Hsieh, C.-T., Yamazaki, H., Wang, J., Kamitani, T., Yamamoto, Y., \& Fukuhara, S. (2021). Quality of life and disability-free survival in the elderly: The Locomotive Syndrome and Health Outcome in Aizu Cohort Study. Journal of Aging and Health, 33(3-4), 197-204.

11. Ul-Haq, Z., Mackay, D. F., \& Pell, J. P. (2014). Association between physical and mental health-related quality of life and adverse outcomes a retrospective cohort study of 5,272 Scottish adults. BMC Public Health, 14, 1197.

12. Phyo, A. Z. Z., Ryan, J., Gonzalez-Chica, D. A., Woods, R. L., Reid, C. M., Nelson, M. R., et al. (2021). Health-related quality of life and all-cause mortality among older healthy individuals in Australia and the United States: A prospective cohort study. Quality of Life Research, 30(4), 1037-1048.

13. Phyo, A. Z. Z., Ryan, J., Gonzalez-Chica, D. A., Stocks, N. P., Reid, C. M., Tonkin, A. M., et al. (2021). Health-related quality of life and incident cardiovascular disease events in communitydwelling older people: A prospective cohort study. International Journal of Cardiology. https://doi.org/10.1016/j.ijcard.2021.07. 004

14. Sajobi, T. T., Wang, M., Awosoga, O., Santana, M., Southern, D., Liang, Z., et al. (2018). Trajectories of health-related quality of life in coronary artery disease. Circulation: Cardiovascular Quality and Outcomes, 11(3), e003661.

15. Park, J.-H., Jung, Y. S., Kim, J. Y., Jo, Y., \& Bae, S. H. (2020). Trajectories of health-related quality of life in breast cancer patients. Supportive Care in Cancer, 28(7), 3381-3389.

16. Hardy, S. E., Concato, J., \& Gill, T. M. (2002). Stressful life events among community-living older persons. Journal of General Internal Medicine, 17(11), 841-847.

17. Cherry, K. E., Brown, J. S., Kim, S., \& Jazwinski, S. M. (2016). Social Factors and Healthy Aging: Findings from the Louisiana Healthy Aging Study (LHAS). Kinesiol Rev (Champaign), 5(1), $50-56$.

18. Lue, B.-H., Chen, L.-J., \& Wu, S.-C. (2010). Health, financial stresses, and life satisfaction affecting late-life depression among older adults: A nationwide, longitudinal survey in Taiwan. Archives of Gerontology and Geriatrics, 50, S34-S38.

19. Fernández-Niño, J. A., Bonilla-Tinoco, L. J., Manrique-Espinoza, B. S., Romero-Martínez, M., \& Sosa-Ortiz, A. L. (2018). Work status, retirement, and depression in older adults: An analysis of six countries based on the Study on Global Ageing and Adult Health (SAGE). Social Science and Medicine Population Health, 6, 1-8.

20. Tajvar, M., Arab, M., \& Montazeri, A. (2008). Determinants of health-related quality of life in elderly in Tehran, Iran. $B M C$ Public Health, 8(1), 323.

21. Adams, R. J., Howard, N., Tucker, G., Appleton, S., Taylor, A. W., Chittleborough, C., et al. (2009). Effects of area deprivation on health risks and outcomes: A multilevel, cross-sectional, Australian population study. International Journal of Public Health, 54(3), 183-192.

22. Dai, H., Jia, G., \& Liu, K. (2015). Health-related quality of life and related factors among elderly people in Jinzhou, China: A cross-sectional study. Public Health, 129(6), 667-673.

23. Jalenques, I., Rondepierre, F., Rachez, C., Lauron, S., \& Guiguet-Auclair, C. (2020). Health-related quality of life among community-dwelling people aged 80 years and over: 
A cross-sectional study in France. Health and Quality of Life Outcomes, 18(1), 126.

24. Liu, N., Andrew, N. E., Cadilhac, D. A., Yu, X., Li, Z., Wang, J., et al. (2020). Health-related quality of life among elderly individuals living alone in an urban area of Shaanxi Province, China: A cross-sectional study. Journal of International Medical Research, 48(4), 1-14.

25. Aspree Investigator Group. (2013). Study design of ASPirin in reducing events in the Elderly (ASPREE): A randomized, controlled trial. Contemporary Clinical Trials, 36(2), 555-564.

26. McNeil, J. J., Woods, R. L., Nelson, M. R., Murray, A. M., Reid, C. M., Kirpach, B., et al. (2017). Baseline characteristics of participants in the ASPREE (ASPirin in Reducing Events in the Elderly) study. Journals of Gerontology Series A: Biomedical Sciences and Medical Sciences, 72(11), 1586-1593.

27. McNeil, J., Nelson, M., Woods, R., Lockery, J., Wolfe, R., Reid, C., et al. (2018). Effect of Aspirin on all-cause mortality in the healthy elderly. The New England Journal of Medicine, 379(16), 1519-1528.

28. McNeil, J. J., Woods, R. L., Ward, S. A., Britt, C. J., Lockery, J. E., Beilin, L. J., et al. (2019). Cohort profile: The ASPREE longitudinal study of older persons (ALSOP). International Journal of Epidemiology, 48(4), 1048-1049h.

29. Ware, J. E., Jr., Kosinski, M., \& Keller, S. D. (1996). A 12-item short-form health survey: Construction of scales and preliminary tests of reliability and validity. Medical Care, 34(3), 220-233.

30. Ware, J. E., Jr., Kosinski, M., Turner-Bowker, D. M. \& Gandek, B. (2002). User's manual for the SF-12v2® Health Survey (With a Supplement Documenting SF-12® Health Survey). QualityMetric Incorporated.

31. Australian Bureau of Statistics. (2011). 2033.0.55.001-Census of Population and Housing: Socio-Economic Indexes for Areas (SEIFA). Australian Bureau of Statistics.

32. Australian Bureau of Statistics. (2018). 2033.0.55.001-Census of Population and Housing: Socio-Economic Indexes for Areas (SEIFA), Australia, 2016: The Index of Relative Socio-economic Advantage and Disadvantage (IRSAD). Retrieved April 06, 2021, from https://www.abs.gov.au/ausstats/abs@.nsf/Lookup/by\% 20Subject/2033.0.55.001 2016 Main\%20Features IRSAD 20.

33. Lubben, J. E. (1988). Assessing social networks among elderly populations. Family \& Community Health, 11(3), 42-52.

34. Radloff, L. S. (1977). The CES-D scale: A self-report depression scale for research in the general population. Applied Psychological Measurement, 1(3), 385-401.

35. Stocks, N., González-Chica, D., Woods, R., Lockery, J., Wolfe, R., Murray, A., et al. (2019). Quality of Life for 19,114 participants in the ASPREE (ASPirin in Reducing Events in the Elderly) study and their association with sociodemographic and modifiable lifestyle risk factors. Quality of Life Research, 28(4), 935-946.

36. Australian Government National Health and Medical Research Council. (2019). Draft Australian guidelines to reduce health risks from drinking alcohol. National Health and Medical Research Council.

37. World Health Organization. (2020). Obesity and overweight. Retrieved January 25, 2021, from https://www.who.int/newsroom/fact-sheets/detail/obesity-and-overweight.

38. Asparouhov, T., \& Muthén, B. (2014). Auxiliary variables in mixture modeling: Three-step approaches using Mplus. Structural Equation Modeling: A Multidisciplinary Journal, 21(3), 329-341.

39. Ram, N., \& Grimm, K. J. (2009). Methods and Measures: Growth mixture modeling: A method for identifying differences in longitudinal change among unobserved groups. International Journal of Behavioral Development, 33(6), 565-576.

40. Clark, S. L. \& Muthén, B. (2009). Relating latent class analysis results to variables not included in the analysis. Retrieved 2021,
February 22, from https://www.statmodel.com/download/relat inglca.pdf.

41. Min, D., \& Cho, E. (2018). Patterns in quality of life according to employment among the older adults: The Korean longitudinal study of aging (2008-2014). BMC Public Health, 18(1), 379.

42. Mcmunn, A., Nazroo, J., Wahrendorf, M., Breeze, E., \& Zaninotto, P. (2009). Participation in socially-productive activities, reciprocity and wellbeing in later life: Baseline results in England. Ageing \& Society, 29(5), 765-782.

43. Marques, L. P., Schneider, I. J. C., \& D'orsi, E. (2016). Quality of life and its association with work, the Internet, participation in groups and physical activity among the elderly from the EpiFloripa survey, Florianópolis, Santa Catarina State. Brazil. Cadernos de Saúde Pública, 32(12), e00143615.

44. Ma, X., \& Mcghee, S. M. (2013). A cross-sectional study on socioeconomic status and health-related quality of life among elderly Chinese. British Medical Journal Open, 3(2), e002418.

45. Cattan, M., Hogg, E., \& Hardill, I. (2011). Improving quality of life in ageing populations: What can volunteering do? Maturitas, $70(4), 328-332$.

46. Kahana, E., Kelley-Moore, J., \& Kahana, B. (2012). Proactive aging: A longitudinal study of stress, resources, agency, and well-being in late life. Aging \& Mental Health, 16(4), 438-451.

47. Eriksson, E., Wejåker, M., Danhard, A., Nilsson, A., \& Kristofferzon, M.-L. (2019). Living with a spouse with chronic illness the challenge of balancing demands and resources. BMC Public Health, 19(1), 422.

48. Tan, S. S., Fierloos, I. N., Zhang, X., Koppelaar, E., AlhambraBorras, T., Rentoumis, T., et al. (2020). The association between loneliness and health related quality of life (HR-QoL) among community-dwelling older citizens. International Journal of Environmental Research and Public Health, 17(2), 600.

49. Jakobsson, U., \& Hallberg, I. R. (2005). Loneliness, fear, and quality of life among elderly in Sweden: A gender perspective. Aging Clinical and Experimental Research, 17(6), 494-501.

50. Ong, A. D., Uchino, B. N., \& Wethington, E. (2016). Loneliness and health in older adults: A mini-review and synthesis. Gerontology, 62(4), 443-449.

51. Niedzwiedz, C. L., Richardson, E. A., Tunstall, H., Shortt, N. K., Mitchell, R. J., \& Pearce, J. R. (2016). The relationship between wealth and loneliness among older people across Europe: Is social participation protective? Preventive Medicine, 91, 24-31.

52. Fernandez, A., Garcia-Alonso, J., Royo-Pastor, C., Garrell-Corbera, I., Rengel-Chica, J., Agudo-Ugena, J., et al. (2015). Effects of the economic crisis and social support on health-related quality of life: First wave of a longitudinal study in Spain. British Journal of General Practice, 65(632), e198-e203.

53. Kim, J., \& Lee, J.-E. (2018). Social support and health-related quality of life among elderly individuals living alone in South Korea: A cross-sectional study. Journal of Nursing Research, 26(5), 316-323.

54. De Belvis, A. G., Avolio, M., Spagnolo, A., Damiani, G., Sicuro, L., Cicchetti, A., et al. (2008). Factors associated with healthrelated quality of life: The role of social relationships among the elderly in an Italian region. Public Health, 122(8), 784-793.

55. Jaspers, L., Colpani, V., Chaker, L., Van Der Lee, S. J., Muka, T., Imo, D., et al. (2015). The global impact of non-communicable diseases on households and impoverishment: A systematic review. European Journal of Epidemiology, 30(3), 163-188.

56. Rees, J., O'boyle, C., \& Macdonagh, R. (2001). Quality of life: Impact of chronic illness on the partner. Journal of the Royal Society of Medicine, 94(11), 563-566.

57. Murphy, A., Mcgowan, C., Mckee, M., Suhrcke, M., \& Hanson, K. (2019). Coping with healthcare costs for chronic illness in 
low-income and middleincome countries: a systematic literature review. BMJ Global Health, 4, e001475.

58. Bratt, A. S., Stenström, U., \& Rennemark, M. (2018). Exploring the most important negative life events in older adults bereaved of child, spouse, or both. Omega (Westport), 76(3), 227-236.

59. Utz, R. L., Caserta, M., \& Lund, D. (2012). Grief, depressive symptoms, and physical health among recently bereaved spouses. The Gerontologist, 52(4), 460-471.

60. Ong, A. D., Fuller-Rowell, T. E., \& Bonanno, G. A. (2010). Prospective predictors of positive emotions following spousal loss. Psychology and Aging, 25(3), 653-660.

61. Bratt, A. S., Stenström, U., \& Rennemark, M. (2017). Effects on life satisfaction of older adults after child and spouse bereavement. Aging \& Mental Health, 21(6), 602-608.

62. Li, Y. (2007). Recovering from spousal bereavement in later life : Does volunteer participation play a role? The Journals of Gerontology: Series B, Psychological sciences and social sciences, 62(4), S257-S266.

63. Australian Government \& Department of Social Services. (2020). Seniors. Retrieved August 25, 2021, from https://www.dss.gov.au/ seniors.

64. World Health Organization. (2021). Coronavirus disease (COVID19) advice for the public. Retrieved August 28, 2021, from https:// www.who.int/emergencies/diseases/novel-coronavirus-2019/ advice-for-public.

65. Geirdal, A. Ø., Ruffolo, M., Leung, J., Thygesen, H., Price, D., Bonsaksen, T., et al. (2021). Mental health, quality of life, wellbeing, loneliness and use of social media in a time of social distancing during the COVID-19 outbreak. A cross-country comparative study. Journal of Mental Health, 30(2), 148-155.

66. Victor, C., Grenade, L., \& Boldy, D. (2005). Measuring loneliness in later life: A comparison of differing measures. Reviews in Clinical Gerontology, 15(1), 63-70.

67. Karania, V. K. (2020). Age UK: Measuring the Prevalence of Loneliness in England. Retrieved August 24, 2021, from https:// www.ageuk.org.uk/globalassets/age-uk/documents/reports-andpublications/reports-and-briefings/loneliness/loneliness-measu re-for-england.pdf.

68. Campaign to End Loneliness. (2014). Measuring your impact on loneliness in later life. Campaign to End Loneliness.

Publisher's Note Springer Nature remains neutral with regard to jurisdictional claims in published maps and institutional affiliations. 